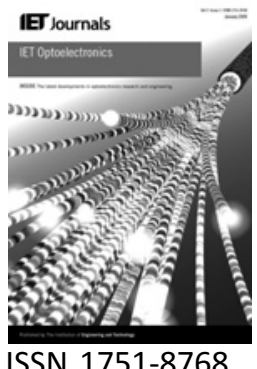

\title{
Spectral response modelling of GaAs-based heterojunction phototransistors for short wavelength detection
}

\author{
H.A. Khan A.A. Rezazadeh
}

Microwave and Communication Systems Research Group, School of Electrical and Electronic Engineering, University of Manchester, Manchester M60 1QD, UK

E-mail: hassan.khan@postgrad.manchester.ac.uk

\begin{abstract}
Spectral response (SR) and optical characteristics of GaAs-based heterojunction phototransistors (HPTs) have been successfully predicted for the first time through an advanced absorption model presented in the present article. The model is based on the accurate prediction of photocarriers in the active layers of the phototransistor which, when related to the base current of transistor in forward active mode, enables the prediction of optical characteristics. The importance of collection efficiency in accurate SR modelling is highlighted and it is not considered unity like all the previous studies on HPTs. The layer dependence of the optical power absorption profile at near-bandgap wavelengths is also investigated and its generalisation as a single exponential has been refuted for GaAs-based HPTs. The measured results at 635, 780 and $850 \mathrm{~nm}$ show good agreement to the predicted results, validating the proposed theoretical model.
\end{abstract}

\section{Introduction}

Particular interest has been shown in heterojunction phototransistors (HPTs), in the last couple of decades, especially for their use in the front-end of high-speed optoelectronic monolithic microwave/millimetre-wave integrated circuit (OEMMIC) photoreceivers [1-3]. OEMMIC technology has reduced the chip size and in turn the cost, while enhancing functionality and performance of the photoreceivers [4]. The process and the layer compatibility of devices is the key for efficient monolithic integration [5]. The performance of HPTs in OEMMIC photoreceivers is supported by their intrinsic or internal current gain (not present in $\mathrm{p}-\mathrm{i}-\mathrm{n}$ photodiodes) [6]. In addition, HPTs do not exhibit extra noise, which is present in high-gain avalanche photodiodes [7]. de Barros et al. [8] showed that even high-speed field effect transistors are limited to a photonic bandwidth of a few megahertz, if photodetection and amplification are to be simultaneously achieved.

Despite the numerous advantages, HPTs have yet to realise their very high potential in optoelectronic integrated circuits. This is mainly because of the fact that in monolithic integration, HPTs share epitaxial layers with heterojunction bipolar transistors (HBTs) and the inevitable design tradeoffs degrade the receiver performance [5]. Hence, there is a need to critically analyse several important parameters of HPTs, which can further revolutionise their use and extend their range of functionality from ordinary sensors to highspeed optical networks.

A key performance parameter of HPTs is their spectral response (SR), which is critical in their usage in optical applications. The modelling of SR for photodiodes has been extensively reported [9-12]; however, several attempts at modelling of SR of HPTs have been limited to a relative or normalised response $[8,13,14]$. The lack of success in the modelling of absolute SR is related to some simplistic assumptions taken from photodiodes and applying these to HPTs. For example, collection efficiency $\left(\eta_{\mathrm{c}}\right)$, which determines the amount of photons absorbed within the active structure of a phototransistor, has been considered unity in all the previous studies $[8,14,15]$, which is not the case. In $\mathrm{p}-\mathrm{i}-\mathrm{n}$ photodiodes, $\eta_{\mathrm{c}}$ can be taken as unity because of a large intrinsic absorption layer. However, in surface-illuminated HPTs, $\eta_{c}$ may not be unity because of 
the limitations in the vertical depth of the absorbing (basecollector depletion) region for efficient transistor action. Material properties, carrier concentrations, temperature of operation and incident photon energy may also affect $\eta_{\mathrm{c}}$, thus affecting the prediction of the spectral response. Hence, a careful analysis of $\eta_{\mathrm{c}}$ is imperative in the accurate modelling of spectral response.

The variations in absorption coefficient $(\alpha)$ with doping also have a significant effect on the optical power absorption profile (PAP) but this is usually not taken into account $[15$, 16]. These variations, in conjunction with different rates of recombination in various layers of transistor can deviate considerably the theoretical results from the experimental. These aspects should be taken into account in the construction of optical PAP of the detectors. Furthermore, subtle variations in refractive index caused by changes in the incident energy should also be incorporated. Other important parameters in characterisation of HPTs are optical gain and noise-equivalent power (NEP). Optical gain incorporates internal gain and the coupling efficiency of the transistor for SR modelling. NEP, along with responsivity, takes into account the leakage currents in phototransistors [17]. A thorough understanding of these parameters is paramount to the correct prediction of optical characteristics of HPTs and will be highly beneficial in the design optimisation of future phototransistors.

In this paper, an advanced optical absorption model for singleHPTs (sHPTs) is proposed. This is then used to predict the SR and optical characteristics of an $\mathrm{N}-\mathrm{p}-\mathrm{n} \mathrm{Al}_{0.3} \mathrm{Ga}_{0.7} \mathrm{As} / \mathrm{GaAs}$ HPT in two-terminal (floating base) mode. The simulated results are compared to measured data at 635, 780 and $850 \mathrm{~nm}$. This generic absorption model should be valid for all material systems (e.g. $\mathrm{InP} / \mathrm{In}_{0.53} \mathrm{Ga}_{0.47} \mathrm{As}$ ) involving HPTs with minor modifications according to the material system.

\section{Methodology}

$\mathrm{SR}$ and optical characteristics of $\mathrm{N}-\mathrm{p}-\mathrm{n} \mathrm{Al}_{0.3} \mathrm{Ga}_{0.7} \mathrm{As} / \mathrm{GaAs}$ HPT have been modelled by accurately predicting the proportion of photogenerated carriers produced by the incident optical signal in the base, collector and sub-collector regions of the device layer (Fig. 1). The photocarriers lost because of recombination have been taken into account [18] and the net photogenerated carriers have then been linked to the input base current for operation of the transistor in forward-active mode. This in turn allows the prediction of optical characteristics and SR of phototransistors. Optical flux absorption and propagation has been analysed in detail, such that the $\eta_{\mathrm{c}}$ and the effect of doping in various layers is incorporated for modelling. MATLAB was used to carry out the simulations.

\subsection{Optical flux absorption}

Optical flux absorption is a material and wavelength-dependent phenomenon. Absorption in the various layers of an HPT

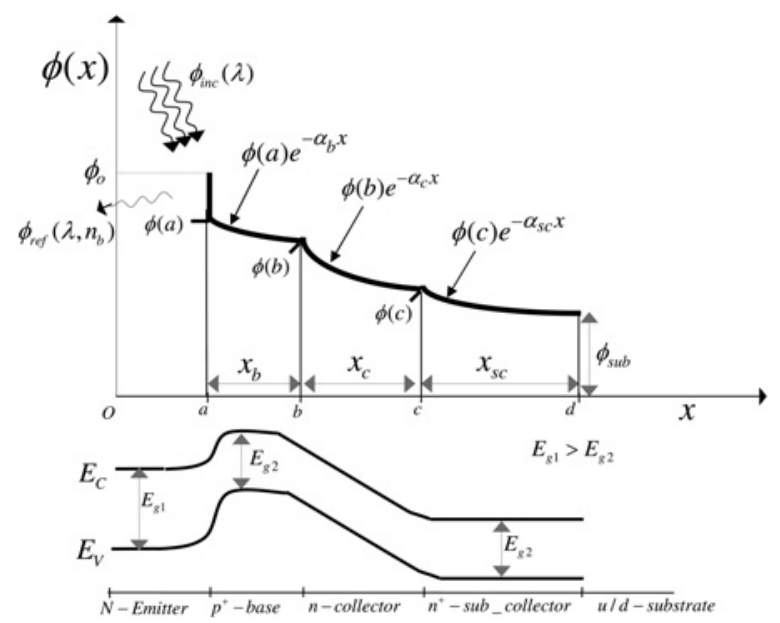

Figure 1 Schematic of optical flux absorption and propagation in various layers of GaAs-based $N-p-n$ HPT along with their schematic energy band diagrams

depends on the bandgap of the material and the wavelength of the incident radiation. The wide bandgap emitter is considered transparent and the incident signal goes unattenuated through the emitter and the absorption starts from the base layer as shown in Fig. 1. Fresnel reflection at the air/emitter interface is irrelevant here as the base of the HPT is illuminated directly. Although the analysis here is performed for a floating-base configuration, the bulk collector is considered depleted because of a large built-in potential between the highly doped p-GaAs base and a low-doped $\mathrm{n}-\mathrm{GaAs}$ collector. From Fig. 1, the optical absorption in the active layers of transistor has been modelled by

$$
\begin{aligned}
\phi_{\mathrm{abs} 1} & =0 \\
\phi_{\mathrm{abs} 2}\left(\lambda, N_{A}\right) & =\phi(a)\left[1-\mathrm{e}^{-x_{\mathrm{b}} \alpha_{\mathrm{b}}}\right] \\
\phi_{\mathrm{abs} 3}\left(\lambda, N_{\mathrm{d}}\right) & =\phi(a)\left[1-\mathrm{e}^{-x_{\mathrm{c}} \alpha_{\mathrm{c}}}\right] \mathrm{e}^{-x_{\mathrm{b}} \alpha_{\mathrm{b}}} \\
\phi_{\mathrm{abs} 4}\left(\lambda, N_{\mathrm{d}}^{+}\right) & =\phi(a)\left[1-\mathrm{e}^{-x_{\mathrm{sc}} \alpha_{\mathrm{sc}}}\right] \mathrm{e}^{-x_{\mathrm{b}} \alpha_{\mathrm{b}}-x_{\mathrm{c}} \alpha_{\mathrm{c}}}
\end{aligned}
$$

where $\phi_{\mathrm{abs} 1}, \phi_{\mathrm{abs} 2}, \phi_{\mathrm{abs} 3}$ and $\phi_{\mathrm{abs} 4}$ are the flux (photons/s) absorbed in the emitter, base, collector and sub-collector of the transistor, respectively. $\alpha_{\mathrm{b}}, \alpha_{\mathrm{c}}$ and $\alpha_{\mathrm{sc}}$ are the absorption coefficients $\left(\mathrm{cm}^{-1}\right)$ for the base, collector and sub-collector layers, respectively. $x_{\mathrm{b}}, x_{\mathrm{c}}$ and $x_{\mathrm{sc}}$ are the widths of the base, collector and sub-collector layers, respectively. $\phi(a)=\phi_{\text {inc }}$ $(\lambda)-\phi_{\text {ref }}\left(\lambda, n_{\mathrm{b}}\right)$ is the value of flux at $x=a, \phi_{\text {inc }}(\lambda)=P_{\text {in }} /$ $E_{\mathrm{ph}}$ is the incident flux radiation and $\phi_{\mathrm{ref}}\left(\lambda, n_{\mathrm{b}}\right)=$ $\phi_{\text {inc }}(\lambda)\left[1-R_{\mathrm{f}}\left(n_{\mathrm{b}}\right)\right]$ is the reflected flux radiation from the base surface. $E_{\mathrm{ph}}=h c / \lambda$ is the incident photon energy, $R_{\mathrm{f}}$ is the Fresnel reflection coefficient, $n_{\mathrm{b}}$ is the refractive index of the GaAs base layer, $c$ is the velocity of light, $b$ is the Planck's constant and $\lambda$ is the wavelength of incident photons.

\subsection{Carrier concentration}

Bandgap variations occur with changes in the doping concentration. This has been taken into account by 
investigating the effect of doping on the absorption coefficient for GaAs. Fig. 2 provides data for absorption coefficients (for all three layers) from 800 to $885 \mathrm{~nm}$ and has been extracted from the published data [19]. However, prior to $800 \mathrm{~nm}$ the effect of doping on absorption coefficient is minimal, which results in the same value of absorption coefficient for base, collector and sub-collector [20].

\subsection{Collection efficiency, $\eta_{c}$}

$\eta_{\mathrm{c}}$ relates the amount of optical flux entering the base layer to the actual amount of useful flux absorbed and this has been modelled by

$$
\eta_{\mathrm{C}}=1-\frac{\phi(d)}{\phi(a)}
$$

where $\phi(a)$ and $\phi(d)$ are the values of flux at $x=a$ and $d$, respectively (Fig. 1). Maximum $\eta_{\mathrm{c}}$ can be achieved if all the flux is absorbed in the base, collector and the subcollector of HPT layers and no photons propagate through to the substrate. However, because of the thickness limitation of the depleted collector layer, some flux may

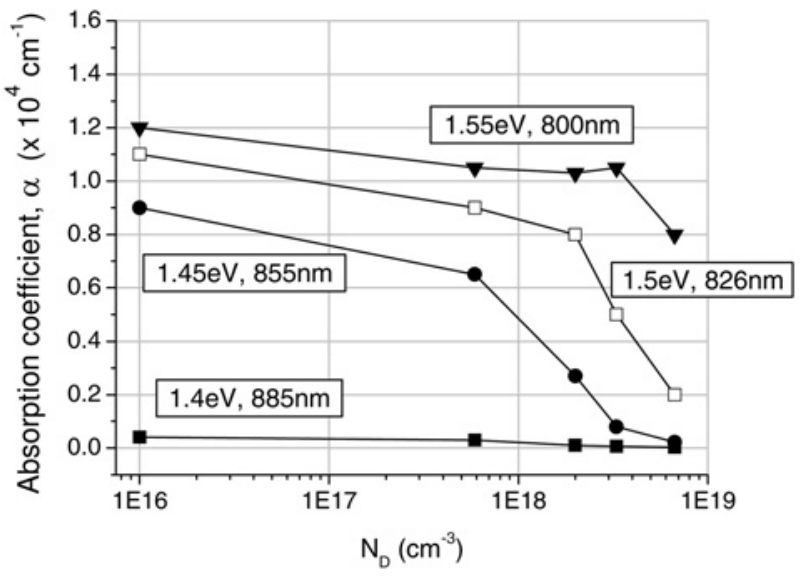

a

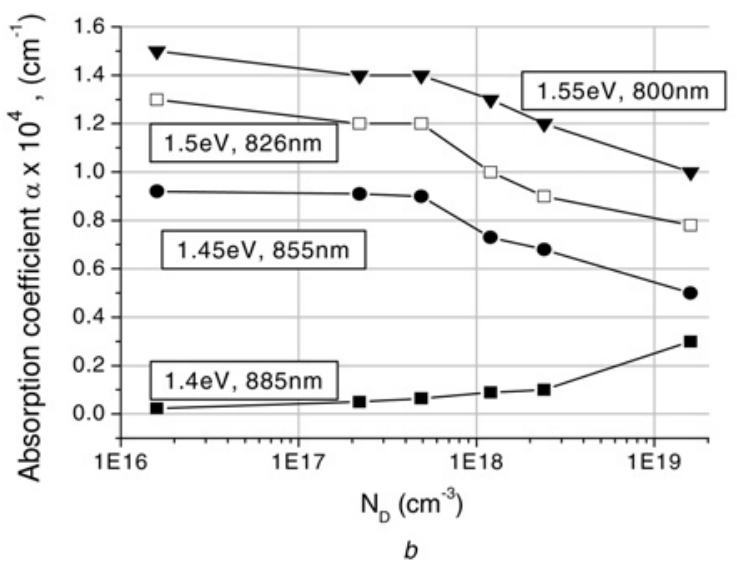

Figure 2 Variation of absorption coefficient, $\alpha$, with different doping concentrations for both

a n- GaAs

$b$ p- [19] propagate through to the highly resistive GaAs substrate. The flux absorbed in the substrate would not contribute towards the photoresponse. This can considerably lower the responsivity, thus making $\eta_{\mathrm{c}}$ a vital parameter in accurate SR prediction.

\subsection{Spectral response, $S R$}

SR specifies the responsivity of a photodetector at every incident wavelength. The responsivity, by definition, is the ratio of photogenerated current $\left(I_{\mathrm{ph}}\right)$ to the incident optical power $\left(P_{\text {in }}\right)$. The first step in the modelling of SR (shown by $R_{\text {spec }}$ in (7)) involves the estimation of total photogenerated current resulting from optical absorption, represented as

$$
I_{\mathrm{ph}}=q \beta \int_{0}^{d}\left(1-R_{\mathrm{f}}\right) \phi_{\mathrm{o}} \alpha \mathrm{e}^{-\alpha x} \mathrm{~d} x
$$

where $\beta$ is the internal current gain of the transistor, $q$ is the charge of electron and $\left(1-R_{\mathrm{f}}\right) \Phi_{\mathrm{o}} \alpha \exp (-\alpha x)$ is the optical generation rate $[11,12]$ in the active layers of the device. So, SR can thus be modelled as

$$
R_{\mathrm{spec}}(\lambda)=\frac{q \beta}{\phi(\mathrm{a}) E_{\mathrm{ph}}(\lambda)} \int_{0}^{d}\left(1-R_{\mathrm{f}}\right) \phi_{\mathrm{o}} \alpha \mathrm{e}^{-\alpha x} \mathrm{~d} x
$$

where $\left(1-R_{\mathrm{f}}\right) \Phi_{\mathrm{o}}$ is the value of input flux at $x=a($ Fig. 1$)$ so it can be represented by $\Phi(a)$ and $\Phi(a) E_{\mathrm{ph}}$ is the input power $\left(P_{\text {in }}\right)$ at the surface base layer. No input flux would be absorbed in the wide bandgap emitter (because of the incident illumination arrangement of the setup) and the flux absorbed in the semi-insulating substrate does not contribute towards the photoresponse and can be ignored in $\mathrm{SR}$ prediction. Therefore (7) can be expanded to

$$
\begin{aligned}
R_{\mathrm{spec}}(\lambda)= & \frac{q \beta}{\phi(\mathrm{a}) E_{\mathrm{ph}}(\lambda)}\left[\alpha_{\mathrm{b}} \phi(\mathrm{a}) \int_{0}^{b-a} \mathrm{e}^{-\alpha_{\mathrm{b}} x} \mathrm{~d} x+\alpha_{\mathrm{c}} \phi(\mathrm{b})\right. \\
& \left.\times \int_{0}^{c-b} \mathrm{e}^{-\alpha_{\mathrm{c}} x} \mathrm{~d} x+\alpha_{\mathrm{sc}} \phi(\mathrm{c}) \int_{0}^{d-c} \mathrm{e}^{-\alpha_{\mathrm{sc}} x} \mathrm{~d} x\right]
\end{aligned}
$$

where $\phi(b)$ and $\phi(c)$ are the values of input flux at $x=b$ and $c$ (Fig. 1).

Optical absorption is a distance-dependent parameter and hence a change in the origin (in the lower-limit of integral) is necessary for accurate prediction of SR. This modification has been proposed to the absorption model presented in our earlier work $[13,14]$, which was only valid for relative $\mathrm{SR}$ estimation. The electron-hole pairs generated from photon absorption in the fully depleted collector layer will be swept across by a strong electric field towards the subcollector and base regions, respectively. Therefore it is assumed that no recombination will occur in the depleted collector. The generation-recombination in the active regions, surface recombination and the leakage currents are considered the same as in the forward-active mode of transistor operation. These effects in the neutral emitter 


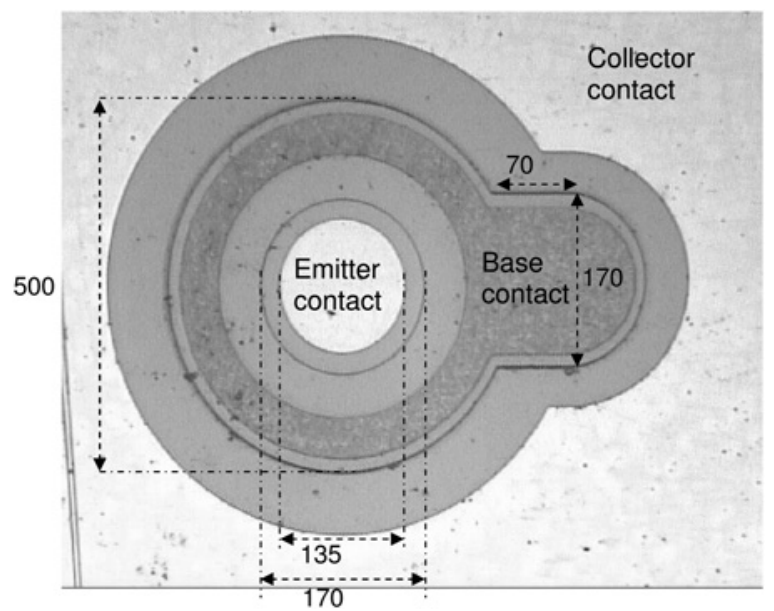

Figure 3 Micrograph of the fabricated $N-p-n A I_{0.3} G_{0.7} A s /$ GaAs HPT

region can also be ignored because hole injection is efficiently suppressed by the use of a wide-bandgap/small-bandgap $(\mathrm{AlGaAs} / \mathrm{GaAs})$ heterostructure. The recombination model [18] for sub-collector takes into account of both Auger and non-radiative (trap-assisted) recombination under the low minority carrier injection approximation (which is applicable for the HPTs in two-terminal configuration). Under these conditions $R_{\text {spec }}$ can be written as

$$
\begin{gathered}
R_{\mathrm{spec}}(\lambda)=\frac{q \beta}{E_{\mathrm{ph}}(\lambda)}\left\{\left(1-\mathrm{e}^{-\alpha_{\mathrm{b}} x_{\mathrm{b}}}\right)+\frac{\phi(b)}{\phi(a)}\left(1-\mathrm{e}^{-\alpha_{\mathrm{b}} x_{\mathrm{b}}}\right)\right. \\
\left.+\frac{\phi(c)}{\phi(a)}\left(1-\frac{\mathrm{e}^{-\alpha_{\mathrm{sc}} x_{\mathrm{sc}}}}{\alpha_{\mathrm{sc}} L_{\mathrm{p}}+1}\right)\right\}
\end{gathered}
$$

where $L_{\mathrm{p}}$ is the hole minority carrier diffusion length in the sub-collector. The photogenerated holes in the base region modify the emitter-base junction for the current to flow similar to the holes injected from the electrical terminal in forward active mode of operation. Therefore the recombination in the base layer has been ignored. The

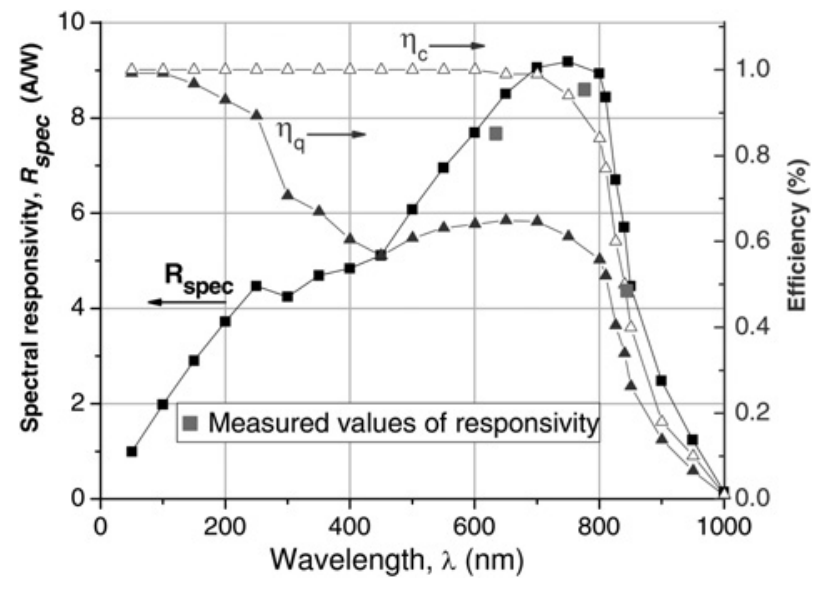

Figure 4 Simulated and measured spectral response for the $\mathrm{Al}_{0.3} \mathrm{Ga}_{0.7} \mathrm{As} / \mathrm{GaAs} \mathrm{HPT}$ at 635,780 and $850 \mathrm{~nm}$ along with its collection, $\eta_{c}$ and quantum efficiency, $\eta_{q}, V_{C E}=2.5 \mathrm{~V}$

predicted SR using (9) is given in Fig. 4 along with the measured results.

\section{Experimental details}

$\mathrm{Al}_{0.3} \mathrm{Ga}_{0.7} \mathrm{As} / \mathrm{GaAs} \mathrm{Npn}$ HPTs with graded base emitter (B-E) junction were grown by MOCVD and large devices were fabricated using standard photolithographic techniques. Transistors had dc current gains of about 23 at collector current of $2 \mathrm{~mA}$. The micrograph of fabricated transistor is shown in Fig. 3. The layer structure is given in Table 1 and the B-C and B-E junction areas are $2.3 \times 10^{5}$ and $2.3 \times 10^{4} \mu \mathrm{m}^{2}$, respectively.

The $\mathrm{Al}_{0.3} \mathrm{Ga}_{0.7} \mathrm{As} / \mathrm{GaAs}$ HPT wafer was set on a four dc-probe station. Three probes were specifically connected to HPT terminal points and the fourth one was connected to optical fibre to illuminate the device base terminal with incident optical radiation. The probes were connected to a Keithley semiconductor parameter analyser for $\mathrm{dc}$ measurements. The LD is aligned to optical fibre $(50 \mu \mathrm{m}$ core diameter) using collimating and focussing lenses and the input current to the laser is driven by a HP 8082A

Table 1 Layer structure of the graded base-emitter $\mathrm{Al}_{0.3} \mathrm{Ga}_{0.7} \mathrm{As} / \mathrm{GaAs}$ Npn transistor

\begin{tabular}{|l|c|c|c|c|c|c|}
\hline \multicolumn{1}{|c|}{ Material } & Mole fraction $x(\%)$ & Thickness $(\mu \mathrm{m})$ & Doping $\left(\mathrm{cm}^{-3}\right)$ & Type & Dopant & Comments \\
\hline $\mathrm{GaAs}$ & - & 0.19 & $5 \times 10^{18}$ & $N$ & $\mathrm{Si}$ & cap layer \\
\hline $\mathrm{Al}_{x} \mathrm{Ga}_{1-x} \mathrm{As}$ & $30-0$ & 0.02 & $5 \times 10^{17}$ & $n$ & $\mathrm{Si}$ & grading layer \\
\hline $\mathrm{Al}_{x} \mathrm{Ga}_{1-x} \mathrm{As}$ & 30 & 0.15 & $5 \times 10^{17}$ & $n$ & $\mathrm{Si}$ & emitter \\
\hline $\mathrm{Al}_{x} \mathrm{Ga}_{1-x} \mathrm{As}$ & $0-30$ & 0.02 & $5 \times 10^{17}$ & $n$ & $\mathrm{Si}$ & grading layer \\
\hline $\mathrm{GaAs}$ & - & 0.09 & $2 \times 10^{19}$ & $p$ & $\mathrm{C}$ & base \\
\hline $\mathrm{GaAs}$ & - & 0.5 & $2 \times 10^{16}$ & $n$ & $\mathrm{Si}$ & collector \\
\hline $\mathrm{GaAs}$ & - & 1.0 & $5 \times 10^{18}$ & $n$ & $\mathrm{Si}$ & sub-collector \\
\hline $\mathrm{GaAs}$ & - & 400 & $\mathrm{U} / \mathrm{D}$ & $\mathrm{S}-\mathrm{I}$ & - & substrate \\
\hline
\end{tabular}


pulse generator. The optical radiation at the end of optical fibre is measured by Anritsu Optical Power Sensor MA 9802A, which is connected to Anritsu Optical Power Meter ML 092A. Further details of the experimental setup can be found elsewhere $[14,21]$.

\section{Results and discussion}

Fig. 4 shows the predicted SR for the $\mathrm{Al}_{0.3} \mathrm{Ga}_{0.7} \mathrm{As} / \mathrm{GaAs}$ HPT along with the modelled collection and quantum efficiencies for the entire short-wavelength spectra. It is constructed by an increment of $50 \mathrm{~nm}$ for high granularity and accurate prediction. The measured values of responsivity at 635,790 and $850 \mathrm{~nm}$ show a very good agreement to the predicted data. The base region of the understudy phototransistor has been illuminated directly and so it is assumed that no input flux gets absorbed in the emitter region. However, if modelling is performed for absorption through transparent contacts and wide bandgap emitter then the responsivity will be lower prior to $680 \mathrm{~nm}$, which corresponds to the band gap of $\mathrm{Al}_{0.3} \mathrm{Ga}_{0.7} \mathrm{As}$ emitter layer $(1.8 \mathrm{eV})$. Material properties such as absorption coefficient and refractive index used for simulation are taken from $[19,20]$. In order to analyse the SR data in Fig. 4, it has been divided into three wavelength regions:

- The first region $(0-350 \mathrm{~nm})$ shows increasing responsivity, $R$, and decreasing $\eta_{\mathrm{q}} \cdot R$ rises with incident flux rate (photons/ s), which increases with wavelength for constant optical power. At the same time the $\eta_{\mathrm{c}}$ tends to unity with photon absorption taking place in the base and collector layers. $\eta_{\mathrm{q}}$ decreases because of the increase in the refractive index of $\mathrm{GaAs}$ with increasing wavelengths [20].

- The second region (350-700 nm) shows rising $R$ and nearly constant $\eta_{\mathrm{q}} . R$ rises as incident flux rate increases and $\eta_{\mathrm{c}}$ tends to unity. $\eta_{\mathrm{q}}$ remains fairly constant because of minimal variation of refractive index in this wavelength region for the $\mathrm{GaAs}$ base layer.

- The third region (700-1000 $\mathrm{nm}$ ) is most significant since it contains the short wavelength of transmission $(850 \mathrm{~nm})$. Theoretically, threshold wavelength for GaAs photodetectors should be around $870 \mathrm{~nm}$. However, it can be seen in Fig. 4 that $R$ and $\eta_{\mathrm{q}}$ start decreasing well before $870 \mathrm{~nm}$ for two reasons. Firstly, the bandgap variation occurs with doping, which lowers the threshold wavelength. This is incorporated by adding the effect of doping on absorption coefficient for GaAs [19]. Secondly and more importantly, $\eta_{c}$ falls significantly at around $775 \mathrm{~nm}$, which causes $R$ and $\eta_{\mathrm{q}}$ to drop.

In order to analyse $\eta_{\mathrm{c}}$, responsivity of every absorbing layer should be taken into account. $\eta_{\mathrm{c}}$ is strictly wavelength and device vertical width-dependent parameter. For the under study device, the vertical depth is constant so the effect of incident wavelength is studied for investigation of $\eta_{\mathrm{c}}$. Two wavelengths, 635 and $850 \mathrm{~nm}$, are used for the analysis of collection efficiency. Fig. 5 shows the current-power
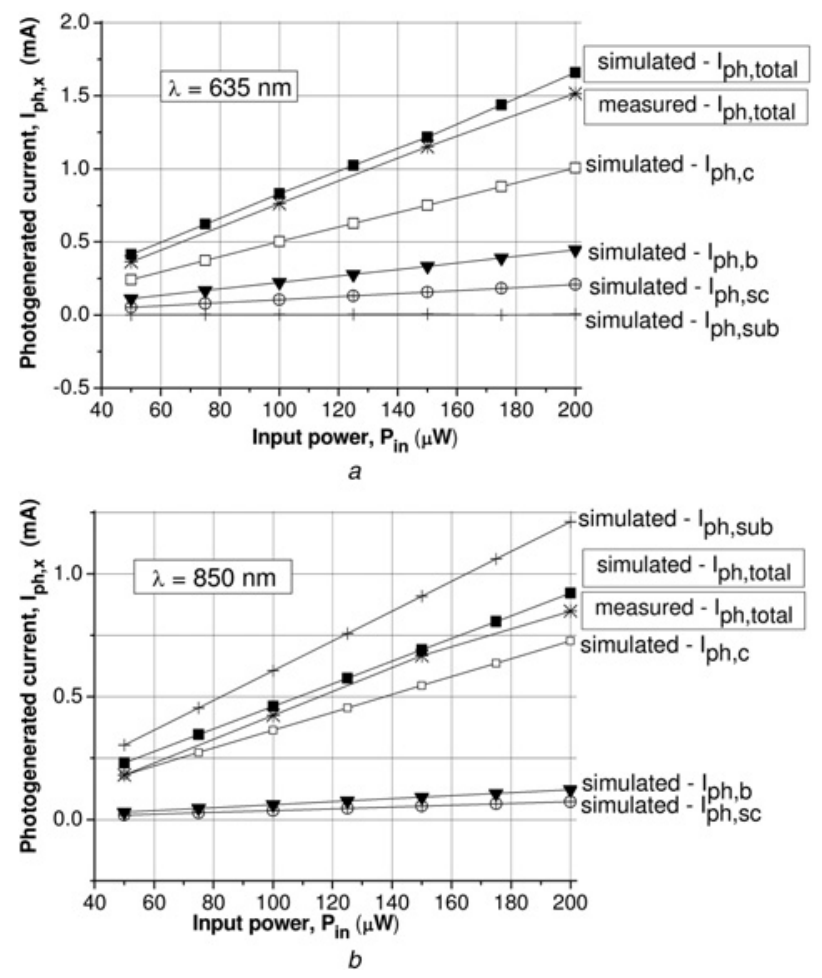

Figure 5 Measured and simulated photogenerated currents with input optical power for various $\mathrm{Al}_{0.3} \mathrm{Ga}_{0.7} \mathrm{As} / \mathrm{GaAs} \mathrm{HPT}$ layers at

a $635 \mathrm{~nm}$

b $850 \mathrm{~nm}\left(V_{\mathrm{CE}}=2.5 \mathrm{~V}\right)$

relationship of each layer at various incident powers. The total photogenerated current $\left(I_{\mathrm{ph}, \text { total }}\right)$ at $635 \mathrm{~nm}$ is higher than that at $850 \mathrm{~nm}$ for the same input power. This is because of the increased photo-absorption in the substrate at $850 \mathrm{~nm}$ which does not contribute towards the photoresponse. In other words, $\eta_{\mathrm{c}}$ at $635 \mathrm{~nm}$ is very close to unity as very small amount of flux propagates through to the substrate resulting in a high responsivity. However, at $850 \mathrm{~nm}, \eta_{\mathrm{c}}$ slumps to less than $50 \%$ as flux absorption in the substrate dominates. Thus, both responsivity and quantum efficiency suffer as a consequence, which is clearly illustrated through Figs. 4 and 5. Collection efficiency has been modelled, in terms of flux propagation through the device, by (5). However, it can also be written in terms of responsivites of each absorbing layer and is modelled as

$$
\eta_{\mathrm{c}}(\lambda)=\frac{R_{\mathrm{b}}(\lambda)+R_{\mathrm{cl} .}(\lambda)+R_{\mathrm{scl} .}(\lambda)}{R_{\mathrm{b}}(\lambda)+R_{\mathrm{cl} .}(\lambda)+R_{\mathrm{scl} .}(\lambda)+R_{\mathrm{sub}}(\lambda)}
$$

where $R_{\mathrm{b}}, R_{\mathrm{cl}}, R_{\mathrm{scl}}$ and $R_{\text {sub }}$ are the responsivity contribution of base, collector, sub-collector and substrate, respectively $\left(R_{\text {sub }}\right.$ not contributing towards the photoresponse). In order to achieve higher collection efficiency and in turn higher responsivity at $850 \mathrm{~nm}$, the device layers should be re-designed. However, this directly affects the performance of the phototransistor and therefore a careful optimisation is necessary to design an optimal phototransistor. This detailed SR analysis thus becomes inevitable for efficient device modelling. 


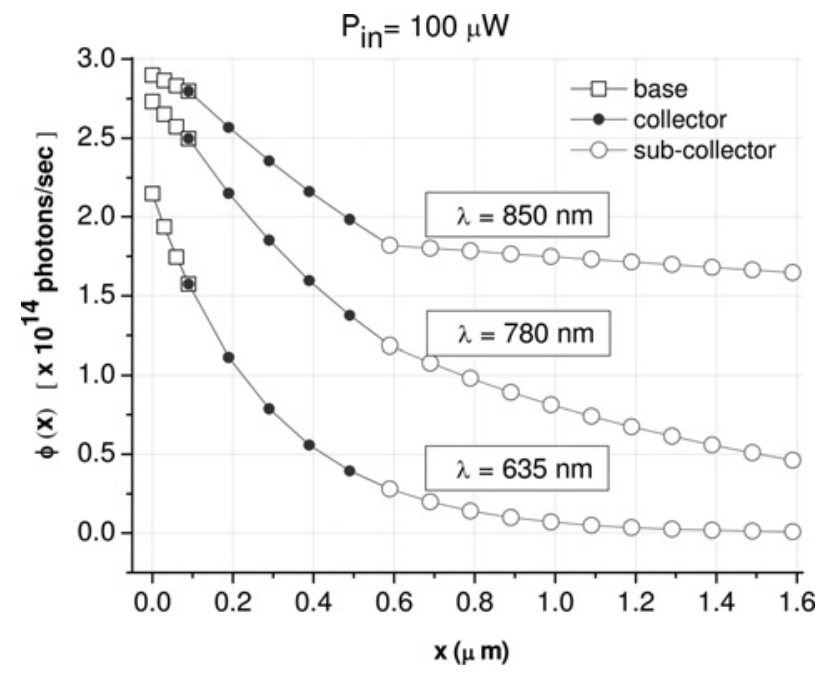

Figure 6 Optical PAP for the $A l_{0.3} G a_{0.7} A s / G a A s$ HPT at three incident wavelengths for incident power of $100 \mu \mathrm{W}$

Optical PAP for the $\mathrm{Al}_{0.3} \mathrm{Ga}_{0.7} \mathrm{As} / \mathrm{GaAs}$ sHPT at 635 , 780 and $850 \mathrm{~nm}$ has been constructed in Fig. 6. The photoabsorption in each layer at $850 \mathrm{~nm}$ varies because of different absorption coefficients and absorption widths of the
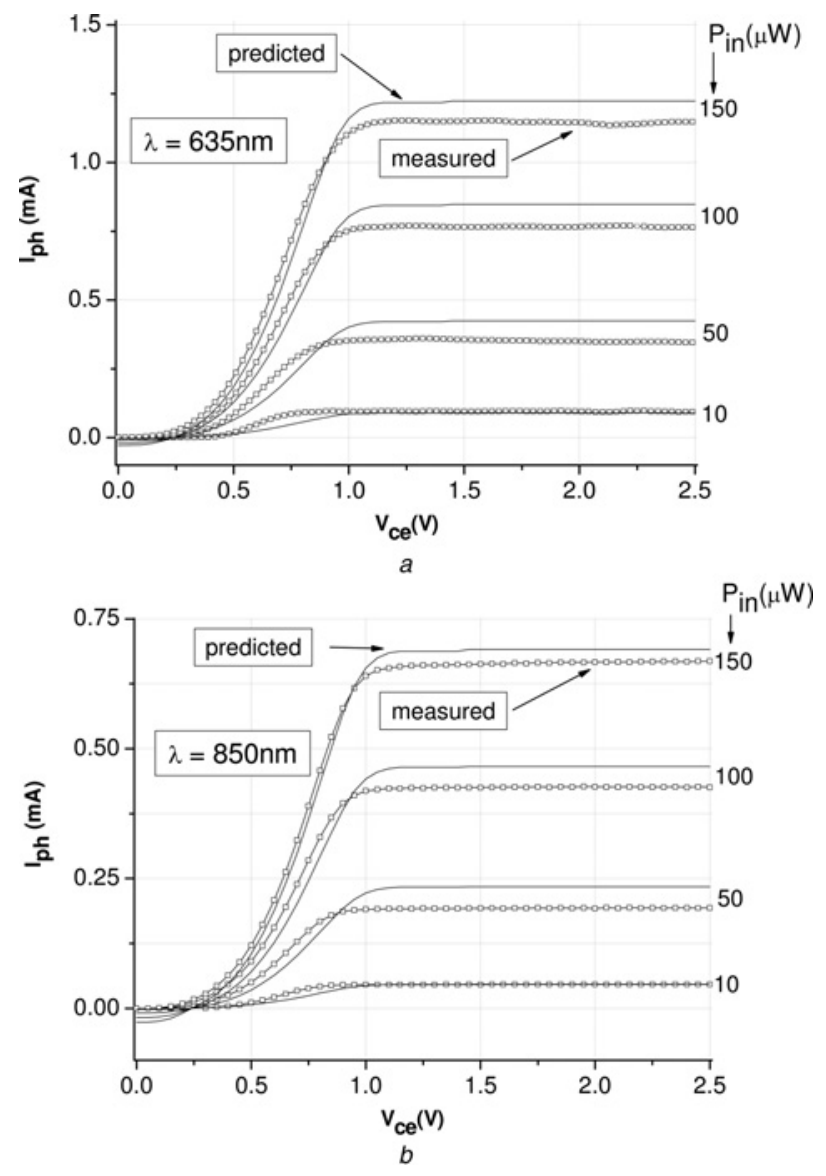

Figure 7 Measured and modelled output characteristics of the $\mathrm{Al}_{0.3} \mathrm{Ga}_{0.7} \mathrm{As} / \mathrm{GaAs}$ HPT at various input optical power for

a $635 \mathrm{~nm}$

b $850 \mathrm{~nm}$ device layers. The rapid absorption in the collector results from a larger absorption coefficient $\left(\alpha_{\mathrm{c}}=8.5 \times 10^{3} \mathrm{~cm}^{-1}\right)$ than that of the base $\left(\alpha_{\mathrm{b}}=5 \times 10^{3} \mathrm{~cm}^{-1}\right)$ and the subcollector $\left(\alpha_{\mathrm{sc}}=0.2 \times 10^{3} \mathrm{~cm}^{-1}\right)$. However, at $635 \mathrm{~nm}$ the absorption coefficient is indifferent of doping and thus can be modelled as a single exponential [19]. At $780 \mathrm{~nm}$, the response tends to shift from single exponential to layerdependent absorption and the variation in $\alpha$ is minute.

Finally, the optical characteristics for the $\mathrm{Al}_{0.3} \mathrm{Ga}_{0.7} \mathrm{As} /$ GaAs HPT at 635 and $850 \mathrm{~nm}$ have been shown in Fig. 7 . A close agreement between the measured and predicted photocurrents is observed as the effect of doping and change in the collection efficiency are incorporated for the absorption model. Some variance between the measured and predicted optical characteristics may be because of slight uncertainty in the coupling of optical fibre to the device.

\section{Conclusions}

The absorption model presented in this paper provides an insight into SR modelling of GaAs-based HPTs for shortwavelength transmission. It was shown that the optical collection efficiency, being strictly geometry and wavelength dependent, should not be considered unity across shortwavelength spectra. A decrease in the collection efficiency for the $\mathrm{Al}_{0.3} \mathrm{Ga}_{0.7} \mathrm{As} / \mathrm{GaAs}$ HPT is observed for wavelengths beyond $700 \mathrm{~nm}$, which has resulted in the smaller values of responsivity. For accurate SR modelling, variations in the absorption coefficient with change in the band gap (because of doping) should be incorporated for the construction of the optical absorption profile. The absorption model and the critical analysis of several important parameters for HPTs presented here can be utilised for performance enhancement through device optimisation in photoreceivers and remote sensing applications employing integrated circuits.

\section{Acknowledgments}

The authors thank Dr. Suba Subramaniam for valuable technical discussions and Sarmad Sohaib for his assistance in MATLAB. Hassan Khan would also like to express his gratitude to Higher Education Commission (HEC) Pakistan for the award of the studentship.

\section{References}

[1] KAMITSUNA H., MATSUOKA Y., YAMAHATA S., SHIGEKAWA N.: 'Ultrahigh-speed InP/InGaAs DHPTs for OEMMICs', IEEE Trans. Microw. Theory Tech., 2001, 49, (10 II), pp. 1921-1925

[2] ABEDIN M.N., TAMER F.S., OLEG V.S., UPENDRA N.: 'AlGaAsSbInGaAsSb HPTs with high optical gain and wide dynamic range', IEEE Trans. Electron Devices, 2004, 51, (12), pp. 2013-2018 
[3] LAI K.-S., HUANG J.-C., HSU K.Y.-J.: 'Design and properties of phototransistor photodetector in standard 0.35-(m SiGe BiCMOS technology', IEEE Trans. Electron. Devices, 2008, 55, (3), pp. 774-781

[4] PEDROTTI K.D., SHENG R.L., NUBLING N.L., FARLEY R.B., CHANG C.W.: 'High-bandwidth OEIC receivers using heterojunction bipolar transistors. Design and demonstration', J. Lightw. Technol., 1993, 11, (10), pp. 1601-1614

[5] LEVEN A., KOPF V., BAEYENS R., CHEN Y.: ‘InP-based doubleheterostructure phototransistors with $135 \mathrm{GHz}$ opticalgain cutoff frequency', Electron. Lett., 2004, 40, (13), pp. $833-834$

[6] KAMITSUNA H.: 'Ultra-wideband monolithic photoreceivers using HBT-compatible HPTs with novel base circuits, and simultaneously integrated with an HBT amplifier', J. Lightw. Technol., 1995, 13, (12), pp. 2301-2307

[7] ChAKRABARTI P., KALRA N.K., AgRAWAL P., GUPTA S.G.: 'Noise modeling of an InP/InGaAs heterojunction bipolar phototransistor', Opt. Eng., 2003, 42, (4), pp. 939-947

[8] DE BARROS L.E.M. JR., PAOLELLA A.F., ROMERO M.Y., HERCZFELD M.J., MADJAR P.R.: 'Photoresponse of microwave transistors to high-frequency modulated lightwave carrier signal', IEEE Trans. Microw. Theory Tech., 1997, 45, (8), pp. $1368-1374$

[9] hanzaZ M.B., MONROY A., MUNOZ E., GIBART E., OMNES P.: 'Modeling of the spectral response of $\mathrm{Al}_{x} \mathrm{Ga}_{1-x} \mathrm{~N}$ p-n junction photodetectors', Eur. Phys. J., Appl. Phys., 2000, 11, (1), pp. 29-34

[10] Bouhdada A.M., VIGUe R., FAURIE F.: 'Modeling of the spectral response of PIN photodetectors impact of exposed zone thickness, surface recombination velocity and trap concentration', Microelectron. Reliab., 2004, 44, (2), pp. $223-228$

[11] GONZALEZ-CUeVAS J.A., TAMER F., ABEDIN M.N.E., HANI E.: 'Modeling of the temperature-dependent spectral response of $\mathrm{In}_{1-x} \mathrm{Ga}_{x} \mathrm{Sb}$ infrared photodetectors', Opt. Eng., 2006, 45, (4), p. 044001
[12] ZHENG H., KANG Y., TANG Y.-W., LI X., FANG J.-X.: 'Study on the spectral response of the Schottky photodetector of GaN', Chin. Phys., 2006, 15, (6), pp. 1325-1329

[13] BASHAR S.A., REZAZADEH A.A.: 'Characterisation of transparent ITO emitter contact InP/InGaAs heterojunction phototransistors'. Proc. Third IEEE Int. Workshop on High Performance Electron Devices for Microwave and Optoelectronic Applications, EDMO 95, 27 November 1995

[14] BASHAR S.A., REZAZADEH A.A.: 'Fabrication and spectral response analysis of $\mathrm{AlGaAs} / \mathrm{GaAs}$ and $\operatorname{InP} / \mathrm{InGaAs}$ HPTs with transparent ITO emitter contacts', IEE Proc.Optoelectron., 1996, 143, (1), pp. 89-93

[15] DE SALleS A.A., HACKBART A.S., SPALDING L.N.: 'A simple model for the GaAs HBT high-frequency performance under optical illumination', Microw. Optic. Technol. Lett., 1994, 7, (9), pp. 392-395

[16] SRIDHARA R.F., ROENKER S.M., PAN K.P., ElLIOTT N.J.: 'Performance enhancement of GalnP/GaAs heterojunction bipolar phototransistors using dc base bias', J. Lightw. Technol., 1998, 16, (6), pp. 1101-1106

[17] KHAN H.A., REZAZADEH A.A., SUbRAMANIAM S.C.: 'Spectral response modelling of heterojunction phototransistors for short wavelength transmission'. Eur. Microwave Conf, Amsterdam, October 2008

[18] CHAND N., HOUSTON P.A., ROBSON P.N.: 'Gain of a heterojunction bipolar phototransistor', IEEE Trans. Electron Devices, 1985, ED-32, (3), pp. 622-627

[19] CASEY H.C.J., SELL D.D., WECHT K.W.: 'Concentration dependence of the absorption coefficient for $\mathrm{n}$ - and $\mathrm{p}$-type GaAs between 1. 3 and 1. 6 EV', J. Appl. Phys., 1975, 46, (1), pp. 250-257

[20] LUSH G.B.: 'Electron lifetimes in p-type GaAs' in BROzEL M.R., STILLMAN G.E. (EDS): 'Properties of Gallium Arsenide' (INSPEC Publications, IEE Press, 1996, 3rd edn.), pp. 87-88

[21] BASHAR S.A.: 'Study of indium tin oxide (ITO) for novel optoelectronic devices' (UMIST, Manchester, 1998), pp. 106-109 